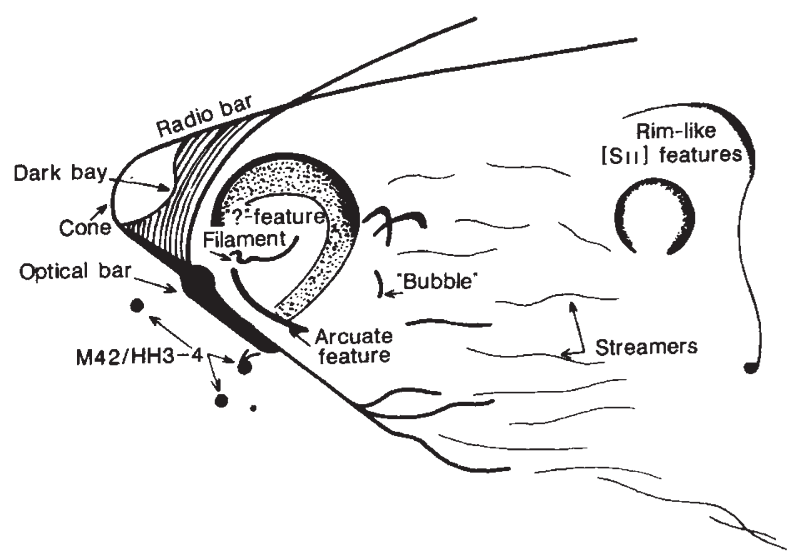

FIG. 2 A schematic view of the Orion Nebula viewed face-on, illustrating some of the prominent structures and features which are present (From F. Yusef-Zadeh Astrophys. J. 361, L19-L22; 1990).

consists of a large envelope (over $10^{5} M_{\odot}$ ) of neutral hydrogen gas $(\mathrm{H} \mathrm{1})$, several large molecular-hydrogen clouds $\left(\mathrm{H}_{2}\right)$ of about $10^{5} M_{\odot}$, H II regions and dark clouds, stretching over almost 20 degrees on the sky. Observations at individual molecular-line wavelengths (J. Bally et al. Astrophys. J. 312, L45-L48; 1987) show that the material is concentrated into large-scale filaments or sheets, containing dense molecular cores in which star formation may be occurring.

At visible wavelengths the outstanding feature is the naked eye-nebula Messier 42 , illuminated by a young group of $\mathrm{OB}$ stars just several million years old, the Trapezium Cluster. These are the brightest members of the I Orion OB stellar association, which contains around 60 O6-B2-class stars, which were probably formed through a sequential starformation mechanism (B.G. Elmegreen \& C.J. Lada Astrophy's. J. 214, 725-741; $1977)$. In this process, the first-generation stars are formed when the edge of a molecular cloud is 'triggered' into gravitational collapse by a violent external event, such as a shock front from a nearby supernova explosion. These first-generation stars may then interact, via stellar winds or radiation pressure, with their placental material, disturbing it and inducing a new generation of star formation.

Observations at optical wavelengths of star-forming regions are often hampered by the absorption and scattering of light by interstellar dust. Most information comes from the molecular-line observations of the neutral gas at millimetre wavelengths, or from radio-wavelength continuum studies of the ionized gas. Yusef-Zadeh made his new radio continuum observations with the Very Large Array (VLA) aperture-synthesis telescope in New Mexico at $20 \mathrm{~cm}$ wavelength. The highangular-resolution $(1.8 \times 1.6$ arcsecond beamsize) images so made cover a relatively large field of view (about one quarter of a square degree), allowing the large-scale morphology of smallscale structure in the gas to be studied.
This is related to the more global mechanisms of star formation.

The radiophotograph (Fig. 1) shows a spectacular network of features centred on the Trapezium cluster. The web-like filamentary structures indicate that stellar winds and ionizing radiation from the central Trapezium Cluster have significantly disturbed the nearby material, creating narrow ionization fronts and strings. The effects of an expanding shell that has resulted from the collision of a stellar wind with material having a strong density gradient will lead to the growth of hydrodynamic Rayleigh-Taylor instabilities in the gas. These cause widespread fragmentation, cooling and the formation of discrete condensations, similar to those reported in the new observations.

On the larger scale, the ionized gas delineates the edge of a cone (Fig. 2), with its apex lying close to an opaque region known as the optical bay. Ionized gas, driven by the internal pressure of the $\mathrm{H}$ II region, appears to be impeded from further expansion by this dense region. The data are consistent with the following picture of star formation in the Orion Nebula. The main area of present-day highmass star formation contains a cluster of about ten $15-25-M_{\odot}$ protostars or premain sequence objects lying close to the front edge of a dense molecular cloud core just behind the centre of the visible nebulosity. Immediately surrounding these stars, gaseous material is subjected to considerable heating and photo-ionization, which excavates a cavity around the star as it clears away this placental material. Outside this, the effects of radiation from the Trapezium cluster give rise to many of the optically visible features of the Orion Nebula, and in particular show how recently formed stars may interact with surrounding material leading to further induced star formation.

The Orion Nebula has a very complex and intricate structure, with many dense filaments, clumps and sheets interspersed with low-density ionized gas, bubbles and cavities. The interaction between the newly formed stars and the surrounding material clearly has a strong influence on the nebula's appearance and evolution. Ultimately star formation will use up or disperse most of the material in the cloud, leaving behind an extensive stellar association, similar to those seen in many other parts of the Galaxy.

Glenn J. White is in the Department of Physics, Queen Mary and Westfield College, Mile End Road, London E1 4NS, UK

\section{That inward eye}

DOCTORS and physiologists frequently need to look inside the human body. All their current methods have drawbacks: X-rays can be damaging, NMR tomography is expensive, ultrasound is cumbersome and not always informative. So why not use light? After all, human tissue is translucent, as can easily be seen by shining a torch through your hand. The snag, of course, is that human tissue scatters light strongly. Simply standing a patient in a light beam like a slide in a projector, would give a hopelessly foggy image.

So Daedalus is refining the idea. He proposes to illuminate the patient, not with continuous light, but with a rapid sequence of sub-picosecond pulses from a modelocked laser. Optically useful, imageforming light will traverse the patient directly, and will emerge first. Scattered light will take a circuitous route, suffering one or more deflections, and will be delayed. By 'time-gating' the emerging light pulses so as to select only their leading edges, a clear visible image should result, free of obscuring scatter. The brevity of the gating time will stretch the art of high speed optical switching to its limits, but the thing looks feasible.

So DREADCO's technicians are scrutinizing naked laser-illuminated volunteers through lithium niobate optical-switch spectacles, while tweaking the gating electronics for maximum subjective transparency. As with X-rays, bones and foreign bodies appear as opaque shadows. Arteries, veins and organs show up according to their effective colour, or fluoresce in the laser light.

By aiming the laser into the patient from the front, and gating the back-reflected light, he could be examined in depth, radar fashion. A fixed time delay between the emission of each light pulse and the brief opening of the optical-shutter spectacles would define a precise out-and-return distance, isolating a narrow slice of the patient's interior for scrutiny. By moving the source back or forward, the slice could be scanned through the patient in a form of optical tomography.

At last the doctor will be freed from the tedious interpretation of screens and photographs. Instead, he will examine and scan through his patient directly. Wearing optical-shutter spectacles and aiming a pulsed laser torch, he will be able to peer at the beating heart, study the movement of a joint or the flexing of a muscle, press on suspect areas to see how the organs beneath respond, check that pills have been correctly swallowed or that an implant is safely in place, select the best approach for surgery, and so on. A patient wearing white cotton or nylon clothes that scatter but hardly absorb light, may not even have to undress.

David Jones 